\title{
AUMENTO DA PREVALENCIA DA ADIPOSIDADE ABDOMINAL EM ESCOLARES BRASILEIROS (2000 - 2015)
}

\section{Pôster}

Autores deste trabalho:

Daniela Casagrande: Faculdade de Medicina de Marília - FAMEMA

Paulo Henrique Waib: Faculdade de Medicina de Marília - FAMEMA

José Augusto Sgarbi: Faculdade de Medicina de Marília - FAMEMA

Área do Trabalho: Pediatria

Número de inscrição: 5101

Data da submissão:18/08/2016 às 09:55

\section{Justificativa}

O aumento da prevalência de sobrepeso e obesidade em crianças e adolescentes é uma preocupação crescente de saúde pública nos países desenvolvidos e em desenvolvimento. No entanto, há poucos estudos com amostras representativas no Brasil.

\section{Objetivo(s)}

Estudar o comportamento do estado nutricional em amostras representativas de adolescentes brasileiros nos últimos 15 anos.

\section{Método(s)}

Em análise transversal investigamos o estado nutricional de adolescentes escolares de ambos os sexos residentes na cidade de Marília, Brasil. Os dados antropométricos e o estado nutricional de 595 escolares do ano de 2000 de foram comparados a 636 do ano de 2015.

\section{Resultado(s)}

Encontramos um aumento significativo da prevalência de obesidade e sobrepeso nos adolescentes avaliados no ano de 2015 comparados aos do ano de 2000 (23,4\% vs. $18,3 \%, \mathrm{p}=0,026)$, mas uma subanálise estratificada por sexo mostrou que esta diferença ocorreu apenas no sexo feminino. Nenhuma diferença significativa foi observada no índice de massa corporal entre os grupos. Por outro lado, a circunferência abdominal (73,5 
cm vs. 77 cm, p <0,001) e a prevalência da adiposidade abdominal (30\% vs. 47,9\%, p $<0,001)$ aumentaram significativamente nos anos de 2015 comparado ao ano de 2000.

\section{Conclusão(ões)}

Os dados mostram um aumento preocupante da prevalência de sobrepeso e obesidade entre adolescentes no Brasil, particularmente no sexo feminino. É alarmante o aumento expressivo da prevalência da adiposidade abdominal em ambos os sexos, em razão de sua associação com aumento do risco cardiometabólico e de mortalidade na vida adulta. 